\title{
Essais
}

Revue interdisciplinaire d'Humanités

Hors-série 7 | 2022

Récits d'outre-thèse

\section{Passer son chemin c'est en prendre un nouveau}

To pass up a path is to take a new one

\section{Michaël Dion}

\section{(2) OpenEdition \\ 12 Journals}

Édition électronique

URL : https://journals.openedition.org/essais/10812

DOI : 10.4000/essais. 10812

ISSN : 2276-0970

Éditeur

École doctorale Montaigne Humanités

\section{Édition imprimée}

Date de publication : 15 avril 2022

ISBN : 979-10-970024-00-0

ISSN : 2417-4211

\section{Référence électronique}

Michaël Dion, « Passer son chemin c'est en prendre un nouveau », Essais [En ligne], Hors-série 7 | 2022, mis en ligne le 01 février 2022, consulté le 18 janvier 2023. URL : http://journals.openedition.org/ essais/10812 ; DOI : https://doi.org/10.4000/essais.10812

Ce document a été généré automatiquement le 18 janvier 2023.

Tous droits réservés 


\title{
Passer son chemin c'est en prendre un nouveau
}

To pass up a path is to take a new one

\author{
Michaël Dion
}

\section{Prologue navrant à la recherche}

1 Il me faut introduire ce récit en narrant ses fondations. En juin 2010, à l'issue d'un master 2 recherche en géographie que je suis en passe de valider, poursuivre mes études me parait une évidence. Ce n'était pas le cas jusqu'alors, mais la présentation de mon mémoire et les encouragements que je reçois me confortentdans cette voie. En tout état de cause, c'est la voie que j'ai logiquement choisie en m'orientant vers une formation ouvrant à la recherche. Une appréhension demeure cependant que je rédige hâtivement mon projet de thèse. En effet, le financement de ma recherche me parait être un préalable indispensable. Je ne compte pas abandonner en chemin faute d'argent, tandis que m'imaginer contraint à m'autofinancer m'effraie. Je candidate par conséquent au «contrat doctoral» de mon laboratoire, mais mon profil n'est pas retenu pour ce financement. Sur cet échec, je quitte l'université. J'y retourne un an plus tard après quelques tergiversations.

2 J'obtiens en 2013 un master 2 recherche en sciences de l'information et de la communication avec la mention très bien. J'ai, durant l'année universitaire, totalement réorienté mes travaux en me découvrant un vif intérêt pour la création contemporaine. Cette appétence est en grande partie née de la rencontre avec le professeur qui deviendra par la suite mon directeur de thèse. Pendant cette dernière année de master, j'ai obtenu un stage au centre d'art contemporain de Bordeaux. Je m'y plais beaucoup. Mon mémoire de fin d'année porte sur le musée en tant qu'espace sensible. Je mets en place ce que j'appelle une "géographie du sensible». Profitant des trois mois que je passe sur place en tant que chargé de communication, je réalise des observations des publics et des œuvres, tandis que je découvre les coulisses des expositions, en 
m'imprégnant des pratiques inhérentes aux processus de création, montage, démontage... À la manière d'un anthropologue, j'observe, je documente et j'analyse les relations public/œuvre en rapport avec les scénographies créées, mettant au jour des comportements, des émotions, des pratiques communicationnelles. Me plaçant du coté du public, l'idée est de considérer le musée comme un terrain d'expérimentation esthétique et sensible. Je m'interroge sur la dimension communicationnelle de ces expériences. Je prends beaucoup de plaisir à mener cette recherche, autant que m'implique mon stage. Dense et recherché, ce travail est très bien reçu.

Fort de mon expérience trois ans auparavant, je suis cette fois convaincu de démarrer un travail de thèse. J'ambitionne toujours un financement. Je candidate en juin au contrat doctoral sans succès. Je ne perds pas espoir car, bénéficiant de très bons rapports avec mon maître de stage au musée, celui-ci m'a proposé de prolonger mon stage de trois mois. J'accepte et débute, dès le mois de septembre, un nouveau stage ; parallèlement je m'inscris en doctorat. Initiant des synergies entre mon laboratoire et le musée, je rédige un projet de thèse visant à s'inscrire dans une Convention Industrielle de Formation par la Recherche (CIFRE). Ce projet porte sur les pratiques numériques dans les espaces d'exposition dédiés à l'art contemporain; il se veut très opérationnel et prospectif, visant à fournir des outils, un état des lieux, des perspectives et des méthodes pour les professionnels œuvrant dans l'institution. Je le conçois spécialement pour cette collaboration, mettant de coté les aspects de mon travail qui pourraient sembler inadéquats dans le cadre d'une CIFRE. J'espère me faire financer par la mairie, de laquelle dépend directement le musée, via la création d'un poste sur une durée de trois ans. Je réunis pour ce faire les soutiens motivés de la direction de l'école doctorale et du laboratoire. Sous le patronage de mon maître de stage, j'initie et j'organise deux réunions entre les directions du laboratoire et du musée. En parallèle, je constitue mon dossier auprès de l'Association Nationale de la Recherche et de la Technologie (ANRT). Les choses semblent correctement s'amorcer. J'obtiens des accords de principe de part et d'autre; mon directeur de thèse me soutient. Quelques amis m'adressent déjà leurs félicitations. Je commence à y croire. Bien mal m'en pris.

4 Le calendrier est relativement serré. Il faut aller vite. Or la direction des ressources humaines de la mairie, reste totalement hermétique à mes sollicitations. Bien qu'ayant fait part de ma situation auprès de la direction du département numérique, je ne parviens pas à faire avancer mon dossier. Pris dans une intense activité de stage et malgré tout relativement confiant, je laisse les choses se décanter : grossière erreur. Subitement, se refusant à toute explication, ne communiquant ni même avec la direction du musée, les ressources humaines et la direction des affaires culturelles de la ville vont clore le dossier. Je n'aurai pas seulement l'opportunité de défendre mon projet ou d'obtenir quelque explication que ce soit. Maintenant je dois terminer ce stage, désappointé. L'ambiance est quelque peu morose, mon maître de stage démissionne peu de temps après la fin de mon stage.

5 Ainsi débutent mes six années de doctorat : elles se bâtissent sur un échec, et sur une forme vive de rancœur. La déception est telle que les premiers mois de recherche sont marqués par un total désinvestissement. Je pensais sincèrement m'épargner mon autofinancement et pouvoir me consacrer totalement à mes études, convaincu que cela me permettrait d'être efficace. Je me connais trop bien, j'angoisse quant à ma capacité à m'impliquer totalement en l'absence du cadre qu'aurait représenté une convention de 
recherche. Avec le recul, force est d'admettre que ce revers a laissé une plaie restée longtemps ouverte. Ayant une confiance somme toute relative en ma capacité à mener à bien les projets inhérents à une forme de carrière, qu'elle quelle soit, cet événement navrant jouera sur mon moral et sur la confiance que je voue en l'institution. Il aura eu sinon le mérite d'éclairer ce qui constitue selon moi la faiblesse majeure du système de la recherche en France, à savoir son incapacité à faire connaître ses valeurs par des structures extérieures. Aucune critique ici des professeurs et professionnels m'ayant accompagné, mais plutôt la révélation d'une faillite du système. Car il existe indéniablement, du côté de la société civile et de ses institutions, une véritable ignorance de la recherche, de l'investissement qu'elle suppose et des bénéfices qu'elle peut engendrer. Je mesure ma responsabilité dans cet échec. Toutefois, je ne suis pas dupe des réalités matérielles, politiques, qui confinent la recherche et son monde, surtout ses étudiants, aux abords de la société. Aujourd'hui, tout cela me parait incompréhensible : pourquoi désinvestir autant sur ce qui constitue le devenir même?

\section{Processus de recherche}

La première année de ma thèse s'avère totalement improductive. Je suis en plein désarroi et j'hésite à abandonner. Durant cette période, je me rends très disponible pour mon travail salarié, dit alimentaire. Je suis à l'époque enquêteur téléphonique. Autrement dit, je fais passer des questionnaires, sondages au téléphone. Vacataire, je peux me rendre indisponible pour mon employeur si je le prévois plus d'une semaine à l'avance. Sinon, je dispose de mon emploi du temps la veille pour le lendemain. Les emplois du temps changent régulièrement; à chaque mission son contrat. Cette organisation du travail a ses avantages comme ses défauts. Avec elle, je peux moduler mes temps de travail ; je suis cependant sous la contrainte des plannings de dernière minute. Je tiens à faire mention de cet emploi salarié car il fut véritablement ce qui me permit de vivre pendant cinq ans, le moyen par lequel j'ai pu faire mes études dans de bonnes conditions. En outre, il devint par moment un espace au sein duquel j'ai fait "camper» ma recherche. Je garde en mémoire ces séquences de travail en double tâche où, entre deux questionnaires, je gratte mes carnets de notes. Dans ce lieu de brouhaha, j'expérimente l'inconfort suprême du bruit, je mets à l'épreuve mon attention et ma concentration, lisant discrètement ouvrages et articles malgré l'interdiction, perdant le fil de ma lecture et de mes pensées dès qu'une personne répond " allô ", le reprenant après m'être assurée qu'elle n'entre pas dans les quotas de mon sondage. De jour en jour, dans ce tumulte que j'exècre sourdement, je prends la mesure du projet dans lequel je me suis lancé. Ici, mes hésitations sont moindres : il me faut mener la recherche à son terme pour accéder à une vie professionnelle plus riche.

7 Le premier pas vers cet objectif consiste en la réécriture globale de mon sujet de thèse. D'une part mon objet d'étude est relativement mal circonscrit, d'autre part il ne correspond plus à mon état d'esprit ou à ce que je veux faire de cette thèse. Sans financement, autant faire en sorte que ce travail soit synonyme de plaisir. Suivant cette résolution, un processus d'émancipation par la recherche se met en œuvre. Dans le souci de faire coïncider sa forme avec ma sensibilité, je choisis de délaisser la dimension opérationnelle prônée jusqu'alors pour faire la part belle à la dimension théorique et à l'expérience subjective. Ces ajustements s'accompagnent d'un travail de terrain poussé pendant trois ans. Le sujet se réoriente vers les thèmes de la création 
contemporaine et de l'exposition. Je conserve comme objet d'étude les pratiques communicationnelles sensibles des visiteurs, mettant en avant leurs caractères anthropologiques. Signe de ma volonté de produire un travail original, je choisis de ne plus seulement considérer le macrocosme muséal pour étudier les pratiques communicationnelles des visiteurs, et focalise mon attention sur un type d'espace, à la fois terrain et forme d'art, beaucoup moins consensuel : l'installation artistique. Choix ambitieux, car, à l'époque, ma connaissance de l'art contemporain se limite plus ou moins à ce que j'en ai découvert pendant mes stages au musée. Je m'appuie intuitivement sur ma formation de géographe pour concevoir ce projet. Partant, je m'attache à traiter les installations artistiques en premier lieu comme des espaces, analogues en certains points à l'espace d'exposition au sein duquel elles se placent, et non seulement comme des œuvres d'art. Ce postulat me confère de l'agilité avec le sujet et me permet de progresser sans risquer de m'enliser dans des problématiques propres à l'histoire de l'art. Guidé par mon directeur de thèse qui s'applique à me canaliser en balisant des zones de recherche pertinentes, la notion de scénographie s'impose rapidement. Elle devient la pierre d'achoppement à partir de laquelle agencer les dimensions communicationnelles hétérogènes que condense l'installation.

Je veux souligner la relation de confiance que je noue pendant ces années avec mon directeur de thèse. Elle m'est essentielle à chaque étape du processus de recherche. Sous sa houlette, j'affronte les pires calvaires méthodologiques, je surmonte mes plus complètes défaillances, tandis que j'effectue mes premiers pas dans mon laboratoire de référence. À l'époque en charge d'un axe d'étude en son sein, il m'incite à prendre part aux activités qui s'y déroulent. C'est dans cet espace de travail, au sein d'une équipe de chercheursréuniemensuellement, que je réalise mes premières communications en rapport avec ma recherche. J'y découvre une parole très riche, ainsi qu'une véritable vie de l'esprit. Ici, je rencontre des interlocuteurs pédagogues, lors de rendez-vous enrichissants. Je comprends également que ce que nous faisons ici est à proprement parler de la recherche. C'est la première fois que je le perçois si nettement. Le programme de réunion de l'axe est conçu pour faire la part belle aux intervenants extérieurs au laboratoire. Ces rencontres ouvrent l'horizon de la recherche : artistes, psychologues, philosophes, chercheurs se succèdent chaque mois pendant deux années. Ces rendez-vous me marquent profondément autant qu'ils nourrissent ma recherche. Plus tard dans mon cursus, les années passant, je participe également aux séminaires que produit l'école doctorale. J'y trouve les meilleures modalités d'enseignement que j'aie jamais expérimentées.

9 J'entame l'écriture au début de la cinquième année. Je mets fin à mon activité salariée car il devient rapidement impossible de passer autant de temps quotidiennement devant un écran d'ordinateur. De plus, l'activité répétitive de mes journées me vide de toute capacité de réflexion le soir venu. Sans revenu, je mets les bouchées doubles pour accélérer un processus d'écriture très fastidieux. Dès le départ apparaissent des lacunes dans ma préparation. Je ne suis clairement pas organisé. S'ensuit des longues périodes de détresse, sans doute communes à tous les doctorants dès lors qu'ils se confrontent au travail d'écriture. Mon professeur, mes collègues, dont certains en sont au même stade que moi, m'apportent le soutien moral nécessaire. Quant à ces derniers, force est d'admettre que leur compagnonnage m'est précieux. Leurs histoires, leurs désirs, leurs volontés, m'offrent le contrechamp nécessaire pour mettre en perspective et nuancer mes déboires personnels. Je mets un an et demi pour boucler l'écriture. 


\section{La recherche aux abords de la société} la thèse «scénographies communicationnelles des installations : regard esthétique et immersion sociale ». Au terme de ma soutenance et malgré les encouragements nourris des membres du jury à mon encontre, j'envisage de prendre mes distances avec la recherche. Je sens que je dois faire au minimum une pause, voire arrêter totalement. Pressentant dans quel état d'esprit je me trouve, c'est à dire euphorique, tout juste libéré, l'un des membres du jury me met en garde : ne pas s'arrêter, continuer tant que le travail d'écriture est bien vivant dans mon esprit, sans quoi cela deviendra de plus en plus difficile de s'y remettre. Sur le moment, je mesure la pertinence de ses conseils, mais au fond de moi le désir de passer à autre chose est à l'œuvre. D'ailleurs, passé le temps du contentement et des vacances, je rechigne à m'y replonger, à publier, à constituer mes dossiers, etc. Ce renoncement est en premier lieux motivé par ma situation financière. Désormais docteur en sciences humaines, j'ai 32 ans, deux enfants, et j'ai épuisé mes droits au chômage. Ma compagne m'entretient depuis plusieurs mois, je ne veux pas continuer le chemin de croix de ces dernières années. Il est hors de question de reprendre la vie d'autrefois mêlant péniblement travail alimentaire, vie de famille et recherche académique. En outre, il faut être réaliste : durant ces six années, je n'ai nullement investi sur ma potentielle carrière dans la recherche. Aucune publication, quelques communications, voilà tout. Le minimum demandé. Ce désinvestissement résonne comme le signe d'un épuisement. Je sais combien il peut être difficile de rester accroché aux wagons; je n'ai pas l'énergie pour me battre. J'ai passé treize ans de ma vie à la faculté en tant qu'étudiant, j'y renonce. Avec le recul, mesurer le temps passé sur ses bancs m'effare. Je n'en suis pas moins heureux d'avoir eu la liberté de puiser dans l'incommensurabilité des ressources qui s'y capitalisent; d'y avoir rencontré des esprits brillants au côté de collègues doctorants parfois très doués, et pour certains bien plus rigoureux que je ne l'étais. Mais ce ne fut pas plus facile pour eux. Je garde en mémoire les difficultés et les oppositions qu'ils rencontrèrent dans l'objectif qui était le leur de faire partie de ce monde, d'obtenir les plus petites responsabilités et finalement de devenir chercheur. Par conséquent, à l'issue de ma soutenance, j'ai le sentiment d'être arrivé au terme d'un parcours. Je me contenterai de cet aboutissement à demi-mot, défaillant quant à ma résilience future dans cet univers pour autant si digne d'intérêt.

Clairement, la recherche et son monde ne me font pas rêver. Pendant ces années, je perçois progressivement comment le déficit global de financement et le manque de moyens affectent toutes ses composantes et limitent très fortement son rayonnement. Ainsi, le laboratoire de recherche, outre cet espace de culture extraordinaire, peut à certains moments recouvrir un climat pernicieux. J'y découvre ainsi des conflits d'intérêts latents, une concurrence exacerbée où chaque équipe se renferme sur ellemême, des collaborations limitées et des situations individuelles problématiques. Par ailleurs, un sentiment sourd m'exaspère progressivement: nous semblons généralement fonctionner en vase clos, entre nous, dans une sorte d'autoréférence épuisante de la recherche à elle-même. Par exemple, je garde en mémoire cette journée de colloque, parmi tant d'autres, sur l'information journalistique. Pendant la pause du matin, il y a cette femme un peu à l'écart, elle téléphone et j'entends son désarroi : elle 
est journaliste. Invitée à intervenir à ce titre, elle semble faire le constat avec son interlocuteur qu'elle est l'unique représente de la profession. Cela semble la désappointer: comment parler de l'information sans faire participer ceuxqui font l'information? Bien sûr, je ne crois pas que la présence de journalistes soit requise pour parler d'information, cependant, j'expose ici par l'exemple l'entre-soi qui me tourmenta. La vérité est que lorsque un laboratoire se confine à pâturer dans le pré borné par la "science", il évite fatalement des occasions potentielles d'enrichir son horizon. C'est pourtant dans ces situations, suffisamment rares pour être soulignées, aux allures de rencontre, que se sont jouées les phases les plus intéressantes de toute ma formation : une pratique en éclairant une autre.

Avec le recul, je déplore l'absence de vision de la part du laboratoire sur son rôle potentiel dans la cité, je regrette le faible nombre d'intervenants étrangers au monde universitaire, je souligne le manque d'ambition et de moyens dévolus à la diffusion des savoir-faire étudiants dans la société civile.

Je sais pertinemment qu'un laboratoire n'est pas un équipement culturel. Et, soyons clair, je ne défends pas le partenariat public/privé pour l'université de demain, bien au contraire, je loue son indépendance et toute démarche qui tend à la lui assurer. J'exècre l'idée de laboratoire sponsorisé, je conspue les lois qui réduisent la recherche à l'idée de projet ou qui valorisent la performance au dépend de la réflexion. Il est absolument certain que le temps du marché n'a rien à voir avec celui de la recherche. Pour autant, force est d'admettre que le monde de la recherche est inaudible auprès du plus grand nombre - surtout lorsqu'il s'agit de recherche fondamentale. C'est un sentiment qui demande du temps pour être appréhendé que celui de toucher un travail extrêmement intéressant et simultanément subir une forme de marginalisation corollaire de la place qu'occupe, ou que manque d'occuper, la recherche en sciences humaines et sociales dans la société contemporaine. À cet égard, j'ai compris avec le temps que la tâche la plus délicate d'un doctorant n'est pas sa recherche mais l'explicitation de sa recherche. Conduire sa recherche, produire son mémoire de thèse, s'accompagne d'un travail de communication omniprésent, que j'ai pour ma part quelque peu sous estimé.

La recherche aux abords de la société, c'est donc ce mouvement double d'une recherche nécessairement en retrait vis-à-vis de l'actualité du monde, d'une certaine manière inactuelle par essence, et d'une société qui manque de familiarité avec elle. Aujourd'hui, le commentaire sur cette forme de discordance s'enrichit par le récit de mes errances professionnelles.

\section{Docteur, et après ?}

Bien que la recherche soit peu ou prou l'unique domaine dans lequel je possède une expérience réelle, je suis convaincu que mon profil pourrait faire recette en dehors de l'université. Les arts visuels, la culture plus généralement, sont des domaines qui m'attirent et que je souhaite pénétrer. Presque naïvement, j'ambitionne de trouver un emploi à la hauteur de ces années passées à me former: un emploi intéressant et correctement rémunéré. Là dessus, je déchante vite. Mon manque d'expérience et de savoir-faire technique me sont clairement reprochés. Les seules expériences viables sont celles que je cumule depuis quelques années en parallèle de ma thèse et que je continue aujourd'hui : bénévolat, activité associative... Là, je me forme en autonomie sur des aspects pratiques nouveaux. Ces activités me permettent de supporter l'idée 
que mon diplôme ne vaut rien aux yeux de la plupart des employeurs. Car, face à eux, je suis tantôt trop diplômé, tantôt trop cher, tantôt inapte à la "réalité " d'unmonde professionnel que je n'ai pas suffisamment expérimenté. J'avoue parfois me questionner passagèrement sur l'intérêt de faire mention de ma thèse lorsque je candidate pour des postes où je me trouve en concurrence avec des personnes de niveau BTS cumulant service civique, bénévolat et voyage Erasmus. Je caricature. Il n'empêche que j'affronte un manque de reconnaissance et une méconnaissance globale de la formation que recouvre le doctorat dans l'ensemble de mes démarches professionnelles. Rares sont les potentiels employeurs qui mesurent combien j'ai expérimenté le travail en autonomie, combien j'ai appris à gérer l'incertitude inhérente à toute forme de projet d'ampleur. Tout cela, c'est le résultat de six années de pérégrination, mise à l'épreuve unique en son genre, et par conséquent, formation d'une grande richesse mais qui manque de transparence pour la majorité. Certaines rencontres, à l'inverse, rendent justice au travail accompli. Je pense en particulier à cet artiste rencontré lorsque j'étais médiateur bénévole sur une installation artistique. Alors que je lui exposais mes problèmes d'insertion sur le marché de l'emploi et remettais en question mes compétences réelles, ce dernier me fit remarquer que j'étais tout de même le spécialiste des "scénographies communicationnelles ». Le fait est qu'à force d'échecs répétés, j'avais presque oublié cette évidence.

16 Je ne me sens pas victime d'un système, j'assume totalement mon parcours, les choix ou le manque de choix forts qui ont conduit à la situation présente. Cependant, les échecs, les déceptions rencontrées, m'ont fait avancer autant que les réussites. Il n'y a eu et n'y aura aucun temps perdu. Avec le recul, je réalise combien le parcours d'une thèse est intimement mêlé à ce que nous sommes, et plus encore, sans que l'on en soit particulièrement conscient, à ce que nous devenons. Le temps long de la thèse emporte avec lui toute la vie, il l'implique intimement dans une métamorphose de l'esprit. Autrement dit, être doctorant c'est un régime de perception dont on ne peut se détacher : tout ce que nous vivons est la recherche. Cette intrication rend partiellement fou. Devenir docteur, c'est revenir de cet état de schizophrénie légère, reprendre contact avec la réalité d'un monde au sein duquel les apparences se suffisent à ellemême.

17 Aujourd'hui, dans cette grande maison de campagne, entouré d'arbres et de champs, j'entame un nouveau chapitre de ma vie. Je ne manque pas de travail dans ce lieu relativement abandonné et isolé. Je vis en plein air, je cultive mon jardin, je répare tant bien que mal tout ce que compte de croulant ou d'abimé la bâtisse et ses dépendances. Ici je suis comme un jardinier, un gardien, à la fois précaire et bourgeois, hippie et plouc. Dans cet espace immense où toute chose porte sur elle l'histoire d'un vécu, j'essaye, j'expérimente sans beaucoup de contrainte. Avec le recul je suis heureux d'avoir pu m'extraire de Bordeaux. J'y vivais depuis plus de dix ans. C'est pour moi encore étonnant de le constater : j'ai renoncé à cette ville, à ce milieu auquel je tenais tant, j'ai quitté sa chaleur. C'était une décision radicale qui a mûri pendant le premier confinement : en à peine deux mois d'enfermement la ville m'a abandonné. Cinq mois plus tard, gardant à vue le paysage qui me fait face, j'ai le sentiment d'avoir reconnecté certains de mes désirs entre eux. Certes je manque d'argent, je suis ici hébergé, je n'ai pas de carrière et je m'inquiète pour mes enfants. Pour autant j'ai le sentiment qu'ici tout est possible ; après tout, ici, il n'y a rien, l'horizon est si vaste. 